\title{
Acoustic Emissions and monitoring bearing health
}

\author{
D. Mba \\ School of Mechanical Engineering, Cranfield University, Cranfield, Beds. MK43 0AL.
}

\begin{abstract}
Acoustic emission (AE) was originally developed for non-destructive testing of static structures, however, over the years its application has been extended to health monitoring of rotating machines and bearings. It offers the advantage of earlier defect detection in comparison to vibration analysis. Current methodologies of applying AE for bearing diagnosis are reviewed. The investigation reported in this paper was centered on the application of standard acoustic emissions (AE) characteristic parameters on a radially loaded bearing. An experimental test-rig was designed to allow seeded defects on the inner and outer race. It is concluded that irrespective of the radial load, rotational speed and high levels of background noise, simple AE parameters such as r.m.s and AE counts provided an indications of bearing defect. In addition to validating already established $\mathrm{AE}$ techniques, this investigation focuses on establishing an appropriate threshold level for AE counts.
\end{abstract}

Keywords: Acoustic emissions, bearing defect diagnosis, condition monitoring

\section{Introduction}

Acoustic emissions (AE) are defined as transient elastic waves generated from a rapid release of strain energy caused by a deformation or damage within or on the surface of a material (Mathews). In this particular research, AE's are defined as the transient elastic waves generated by the interaction of two surfaces that are in relative 
movement to each other. The interaction of surface asperities and impingement of the bearing rollers over the seeded defect on the outer and inner races will result in the generation of Acoustic Emission.

\section{Acoustic emission and bearing defect diagnosis}

Yoshioka et al have shown that AE parameters identified bearing defects before they appeared in the vibration acceleration range whilst Catlin reported AE activity from bearing defects were attributed to four main factors, including random noise generated. It was noted that signals detected in the AE frequency range represented bearing defects rather than other defects such as imbalance, misalignment, looseness and shaft bending.

Tandon et al investigated AE counts and peak amplitudes for an outer race defect using a resonant type transducer. It was concluded that AE counts increased with increasing load and rotational speed. However, it was observed that AE counts could only be used for defect detection when the defect was less than $250 \mu \mathrm{m}$ in diameter, though AE peak amplitude provided an indication of defects irrespective of the defect size. Choudhury et al employed AE for bearing defect identification on various sized bearings and rotational speeds ranging from 500 to $1500 \mathrm{rpm}$. It was observed that $\mathrm{AE}$ counts were low for undamaged bearings, based on a threshold level of 1Volt. In addition, it was observed that $\mathrm{AE}$ counts increased with increasing load and speed for damaged and undamaged bearings. Tan used a variation of the standard AE count parameter in diagnosis of different sized ball bearings. In addition to the difficulty of selecting the most appropriate threshold level for standard AE counts, Tan sited a couple of other drawbacks with the conventional AE count technique, such as the 
dependence on the count value on the signal frequency. Secondly, it was commented that the count rate was indirectly dependent upon the amplitude of the AE pulses.

A clear relationship between the r.m.s level, rotational speed and radial load has been reported. The use of AE counts is dependent on the particular investigation, and, the method of determining the threshold level is at the discretion of the investigator. For this reason, the investigation presented in this paper firstly validates the use of r.m.s for diagnosis, and secondly, ascertains the suitability of AE counts for bearing diagnosis. In addition, selection of the appropriate threshold level is investigated.

\section{Experimental equipment}

A test rig was designed to simulate early stage of bearing defects, see figure 1 . The rig consisted of a motor/gear box unit providing a rotational speed range of between 10 to $4000 \mathrm{rpm}$. Two aligning support bearings, a rubber coupling and a larger support bearing were employed. The test bearing investigated was a split Cooper spherical roller, type 01C/40GR (bore diameter - 40mm, 10 roller elements). This type of bearing was chosen owing to its ability to be disassembled without removing slave bearings, thereby allowing the test bearing to be regularly inspected throughout the test programme. A radial load was applied to the top of the bearing via a hydraulic cylinder ram supported by an ' $H$ ' frame. All attempts were undertaken to ensure the amount of grease within the bearing remained the same. It must be noted that for all tests and simulations, the receiving transducer was cemented with superglue onto the bearing housing, see insert of figure 1 . To ensure even distribution of the couplant across the face of the sensor, a small amount of glue was place in the centre of the intended position of the sensor. The 
sensor was then carefully pressed onto the surface, spreading the couplant uniformly.

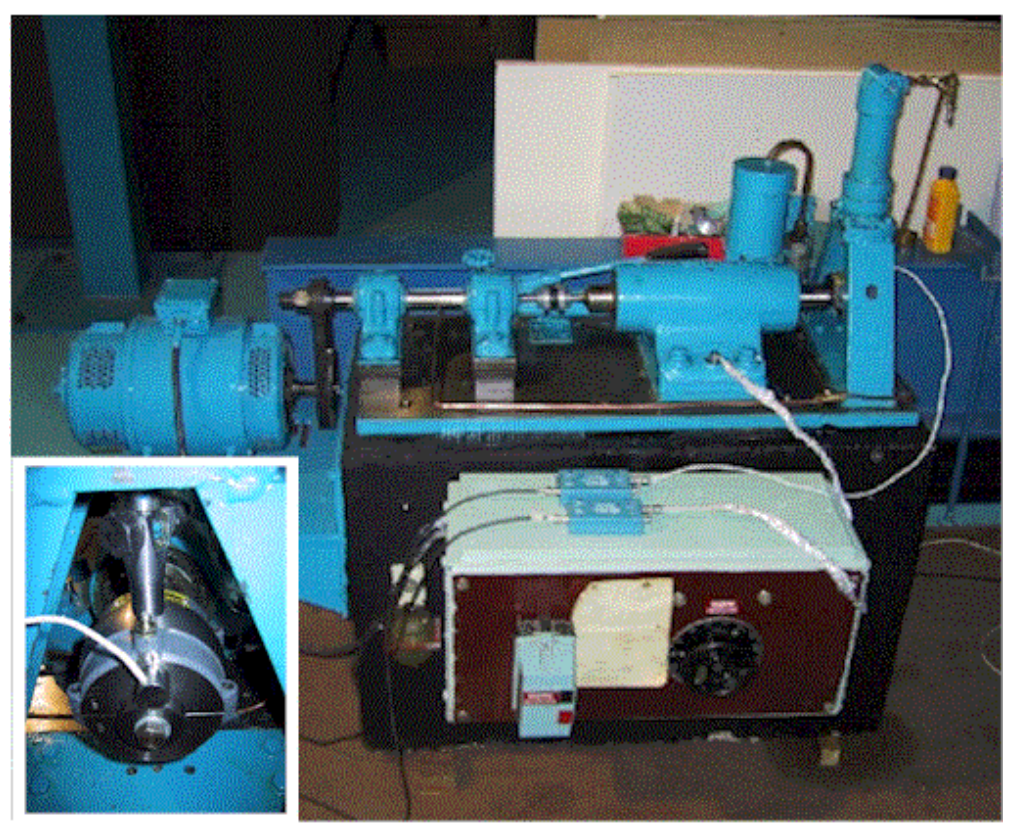

Figure 1 Bearing test-rig; Insert shows close-up of test bearing

\section{Data acquisition system}

A piezoelectric type sensor (Physical Acoustic Corporation type WD) with an operating frequency range of $100 \mathrm{kHz}-1000 \mathrm{kHz}$ was employed. A schematic diagram of the acquisition system is illustrated in figure 2 .

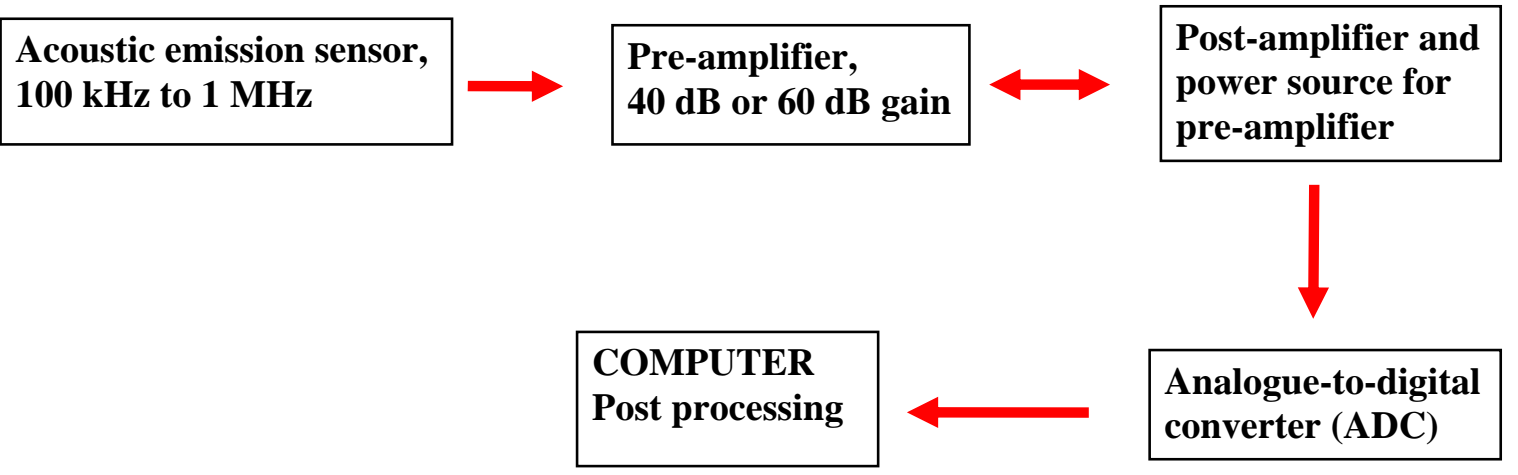

Figure 2 Schematic diagram of acquisition system 
Pre-amplification ranged from 40 to $60 \mathrm{~dB}$ and a total of 33,000 data points were recorded per acquisition (data file) at a sampling rate of $4 \mathrm{MHz}$. One hundred (100) data files were recorded for each simulated case, providing over 0.8 seconds of data per fault simulation. This was equivalent to 8-revolutions of data at 600rpm; 20 revolutions at $1500 \mathrm{rpm}$ and 40 -revolutions at 3000rpm. A trigger level above the electronic noise level was set at 0.5 Volts for data acquisition. The procedure for collecting data simply involved arming the acquisition system at random intervals over a 15-minute period per simulation. It was thought this would determine the robustness of specific AE characteristic parameters for diagnosis of operational bearings.

\section{Data analysis}

The most commonly measured AE parameters are amplitude, r.m.s, energy, counts and events (Mathews). Counts involve counting the number of times the amplitude exceeds a preset voltage (threshold level) in a given time and gives a simple number characteristic of the signal. The r.m.s value gives the intensity of the AE signature. The AE parameters measured for diagnosis in this particular investigation were r.m.s and $\mathrm{AE}$ counts. In determining the threshold level for $\mathrm{AE}$ counts, five values as a percentage of the lowest amplitude background noise observed (600rpm) were employed. The percentage values selected were $10 \%, 30 \%, 50 \%, 70 \%$ and $90 \%$. The reason for selecting these specific values was it offered a wide range of values, particularly useful as the investigators hoped to ascertain and determine the influence of threshold value on AE count results. Usually determining the threshold levels have been at the discretion of the investigator and in most cases, the values are probably 
selected depending on intuition and/or experience on the particular test-rig or machine.

\section{Experimental procedure}

Prior to seeding defects the test-rig was operated to provide an indication of background noise levels. Two types of defects were seeded on the inner and outer races. The seeded fault was a uniform surface line defect that was accomplished with a engraving machine. The nominal width, depth and length of the line defect on the outer and inner race was measured at $1 \mathrm{~mm}, 75 \mu \mathrm{m}$ and $5 \mathrm{~mm}$ for a 'small defect', while the 'large defect' had a length of approximately $15 \mathrm{~mm}$, see figure 3. The test-rig was operated at three different rotational speeds; $600 \mathrm{rpm}, 1500$ rpm and $3000 \mathrm{rpm}$. At each rotational speed three load cases were considered; $0 \mathrm{kN}, 2.4 \mathrm{kN}$ and $4.8 \mathrm{kN}$. For background noise measurements the rig was operated at up to $4000 \mathrm{rpm}$ with no radial load. To simulate realistic diagnostic conditions, the timing of data acquisition for every one of the one hundred data files during test conditions was selected randomly within a 15-minute test period. It was felt that this approach was representative of the method to be employed during diagnosis of operational units. Moreover, it provided a good test on the suitability and robustness of AE for bearing diagnosis. Prior to extracting AE characteristics parameters, all one hundred data files (each of 0.08 seconds duration) were linked, creating a chain equivalent to 0.8 seconds. As such, AE count values calculated for each test condition were in effect an accumulation of counts over one hundred data files and the r.m.s values were equivalent to the average over one hundred data files. 


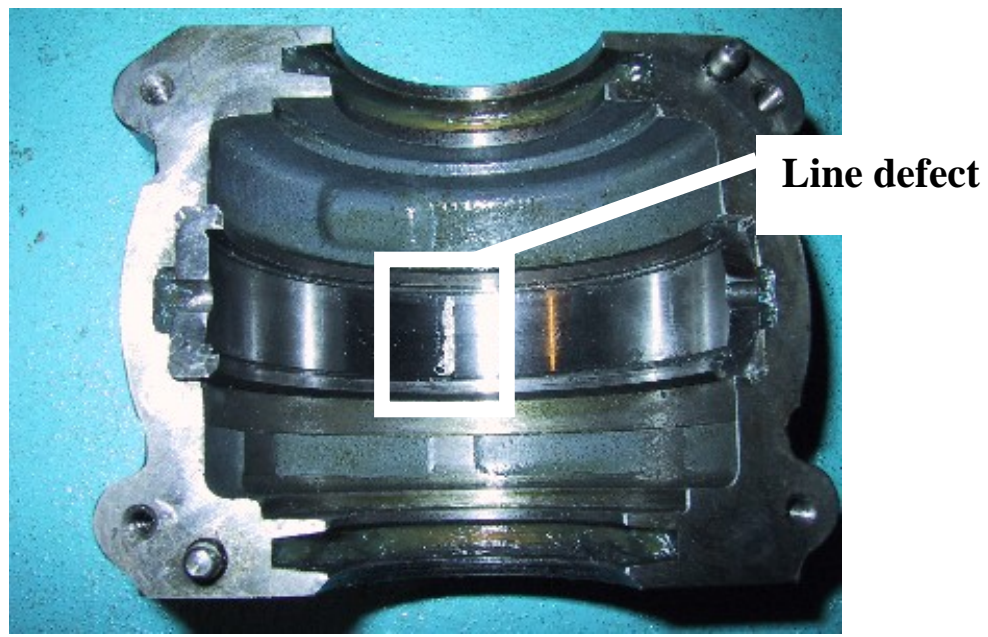

Figure 3 Seeded line defect on outer race

\section{Results for background noise}

It must be noted that at the higher speed (3000 rpm), pre-amplification was reduced to $40 \mathrm{~dB}$. All the results presented are comparative at $60 \mathrm{~dB}$, which implies that a multiplication factor of 10 was applied to all data captured at $40 \mathrm{~dB}$. Prior to analysis all AE signatures were passed through a $5^{\text {th }}$ order median filter (Olli Yli-Harja) to eliminate any spurious electrical spikes.

The following format, with examples, was employed for labeling all AE data presented:

L0; L2; L4; L - load; 0 load value - 0KN; 2 - 2.4KN; 4-4.8KN

N6L0 N - Noise; 6 - speed at $600 \mathrm{rpm}$; L - load; 0 load value - 0KN

Si6, Si15, Si30; S - Small defect; i - inner race; 6;15;30 speed - 600 rpm, 1500 rpm or 3000 rpm. Lo6 - Large defect on the outer race at 600rpm. Li15L2 - Large inner race defect at $1500 \mathrm{rpm}$ and $2.4 \mathrm{KN}$. 
An AE time trace for background noise is shown in figure 4.

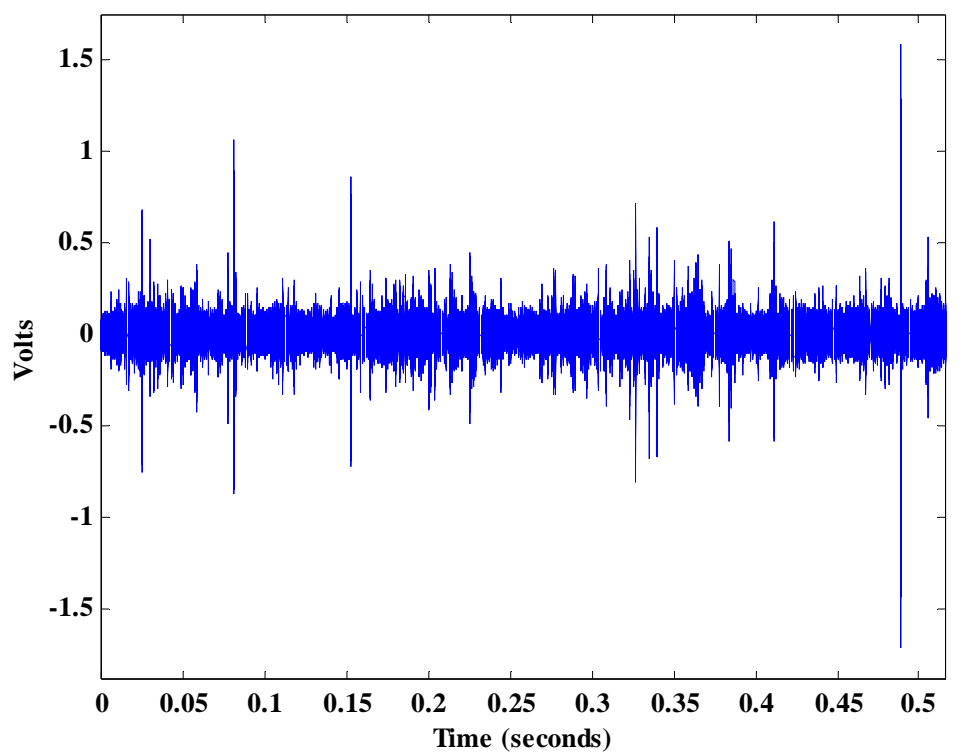

Figure 4 Typical AE time trace background noise; Speed 600rpm, load 0KN.

The AE r.m.s values for background noise at the three different speeds were $0.06 \mathrm{~V}$, $0.37 \mathrm{~V}$ and $0.66 \mathrm{~V}$, at 600,1500 , and $4000 \mathrm{rpm}$ respectively. In addition the lowest maximum amplitude value for all simulation case was 1.7 Volts at $600 \mathrm{rpm}$. As such corresponding threshold values for $\mathrm{AE}$ count analysis were $0.17,0.5,0.85 \mathrm{~V}, 1.2$ and 1.5Volts. Results for AE counts for the specified threshold levels of background noise are presented in figure 5. Clearly $\mathrm{AE}$ counts increased with increasing speed irrespective of the threshold level. 


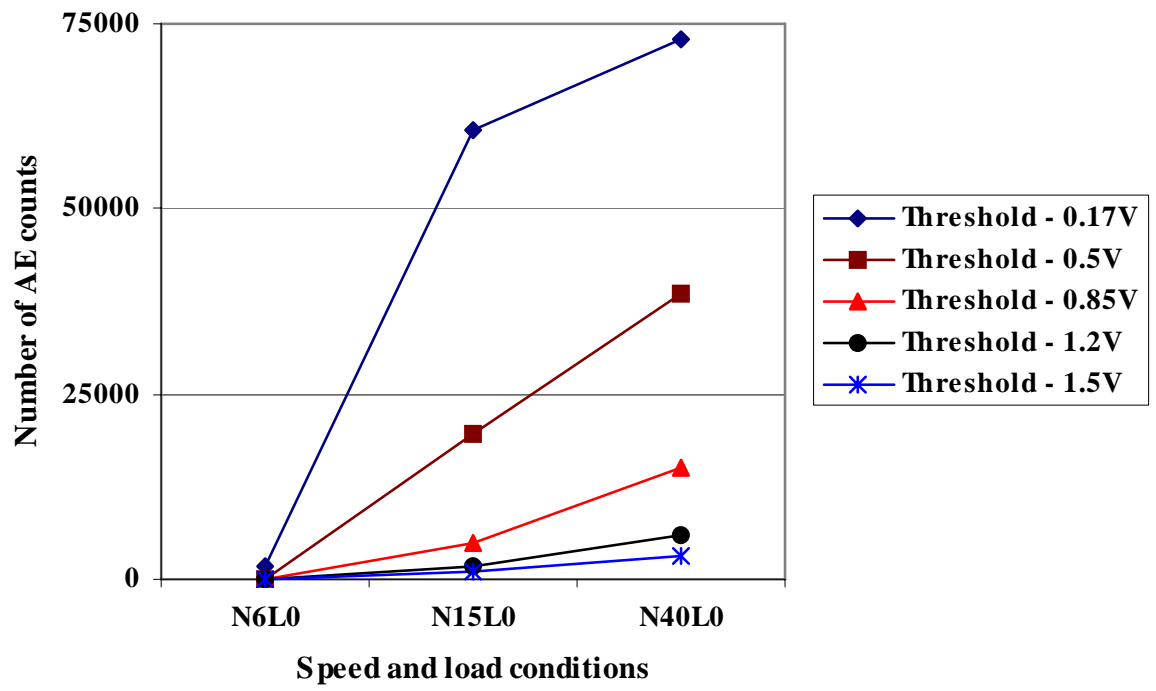

Figure 5 AE counts of background noise

\section{Results for defect simulation}

An AE time trace for an outer race defect is shown in figure 6. Typically, r.m.s values increased with increasing load, speed and defect size, see figure 7. In addition, r.m.s values increased from inner race defects to outer race defects. The difference in r.m.s levels for outer and inner race defects was attributed to attenuation. These results are in agreement with published results of several researchers (Yoshioka et al, Tan, Choudhury et al, and Tandon et al) and forms the basis from which to investigate the influence of threshold levels for AE counts. 


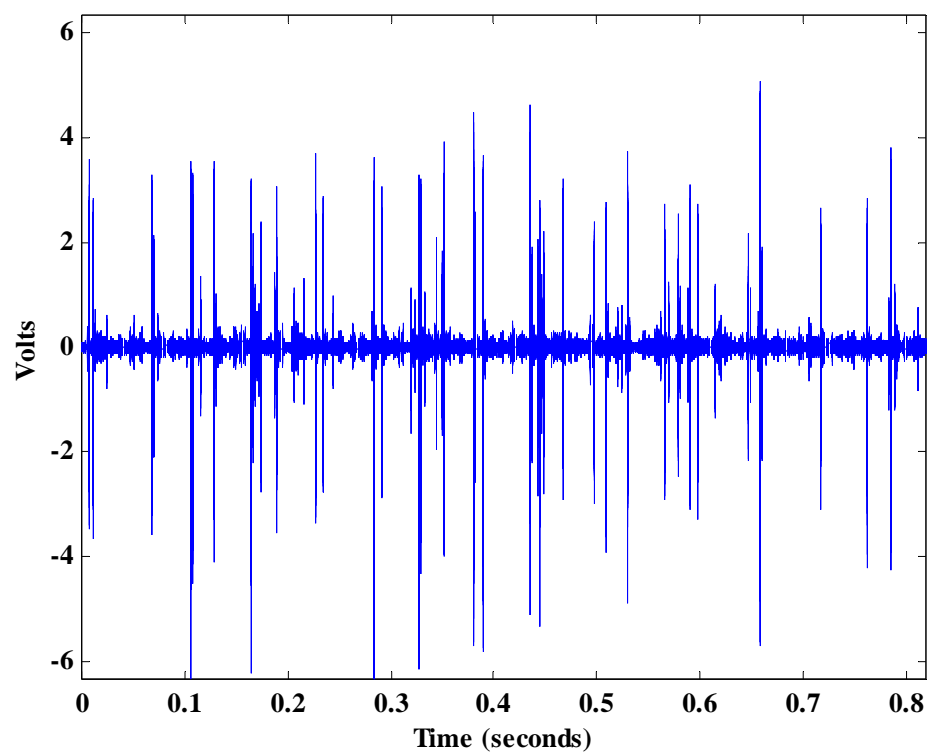

Figure 6 Typical AE time trace for an outer race; Speed 600rpm, load 0KN

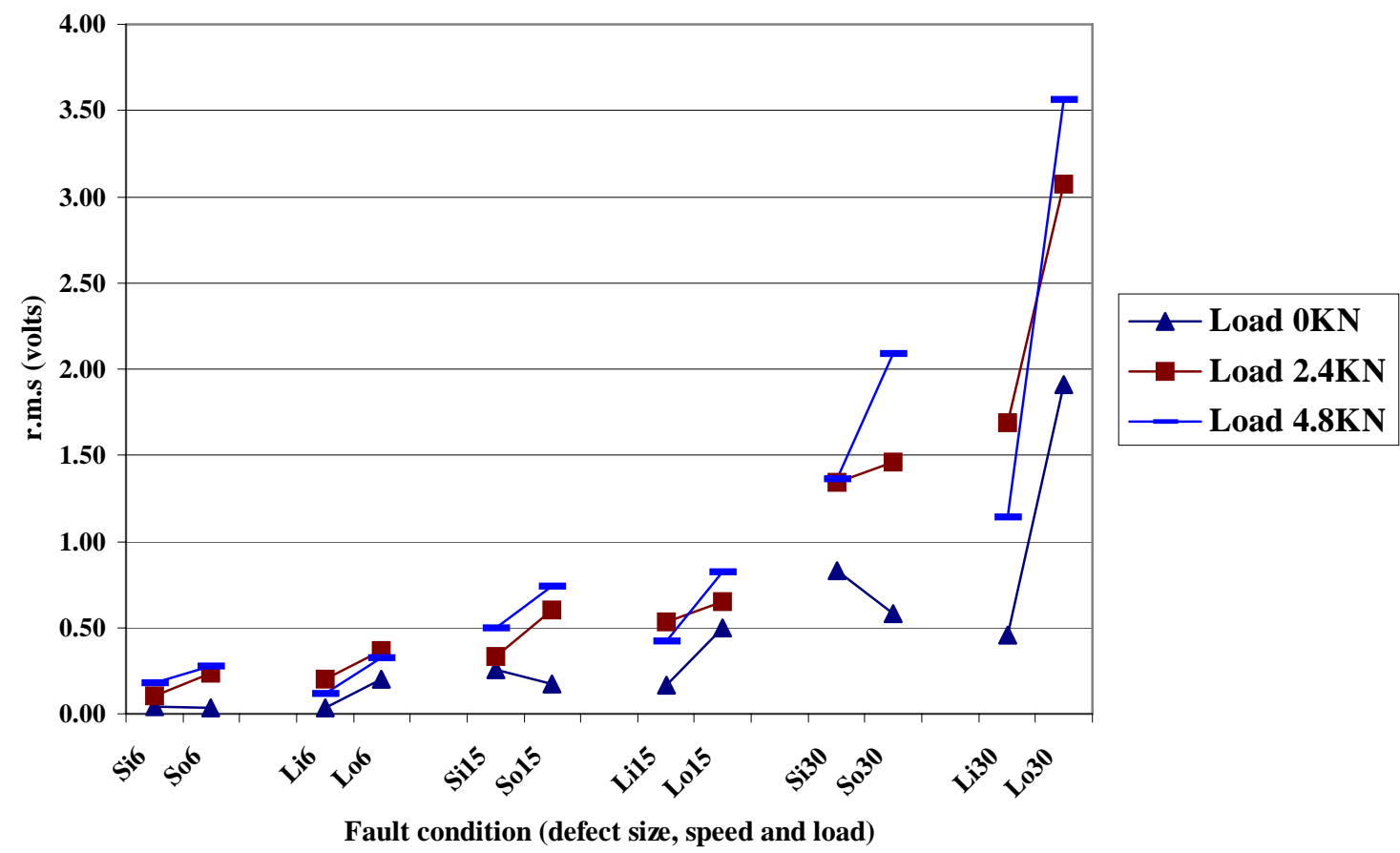

Figure $7 \quad$ r.m.s values for inner and outer race defects as a function of load, speed and defect size

Results of AE counts for all defects at varying speeds and loads can be viewed in figures 8 and 9. Figure 8 shows a general increase in AE counts for increasing 
load at $1500 \mathrm{rpm}$ irrespective of threshold level and defect size. The same trend could be viewed in figure 9 where an increase in AE count with speed and load was observed. These observations also applied at speeds of 600 and 3000rpm.

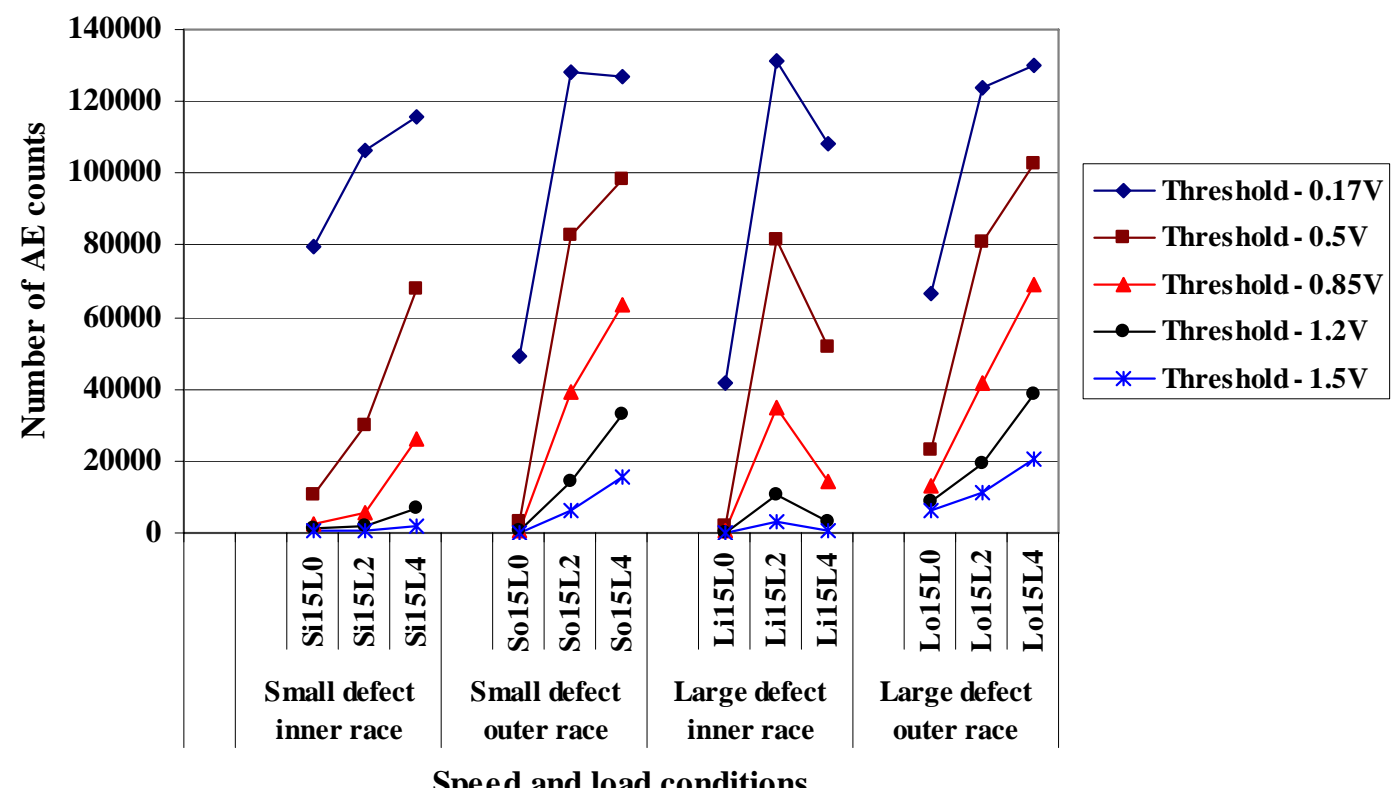

\section{Figure 8 Number of AE counts for outer race defects at 1500 rpm}

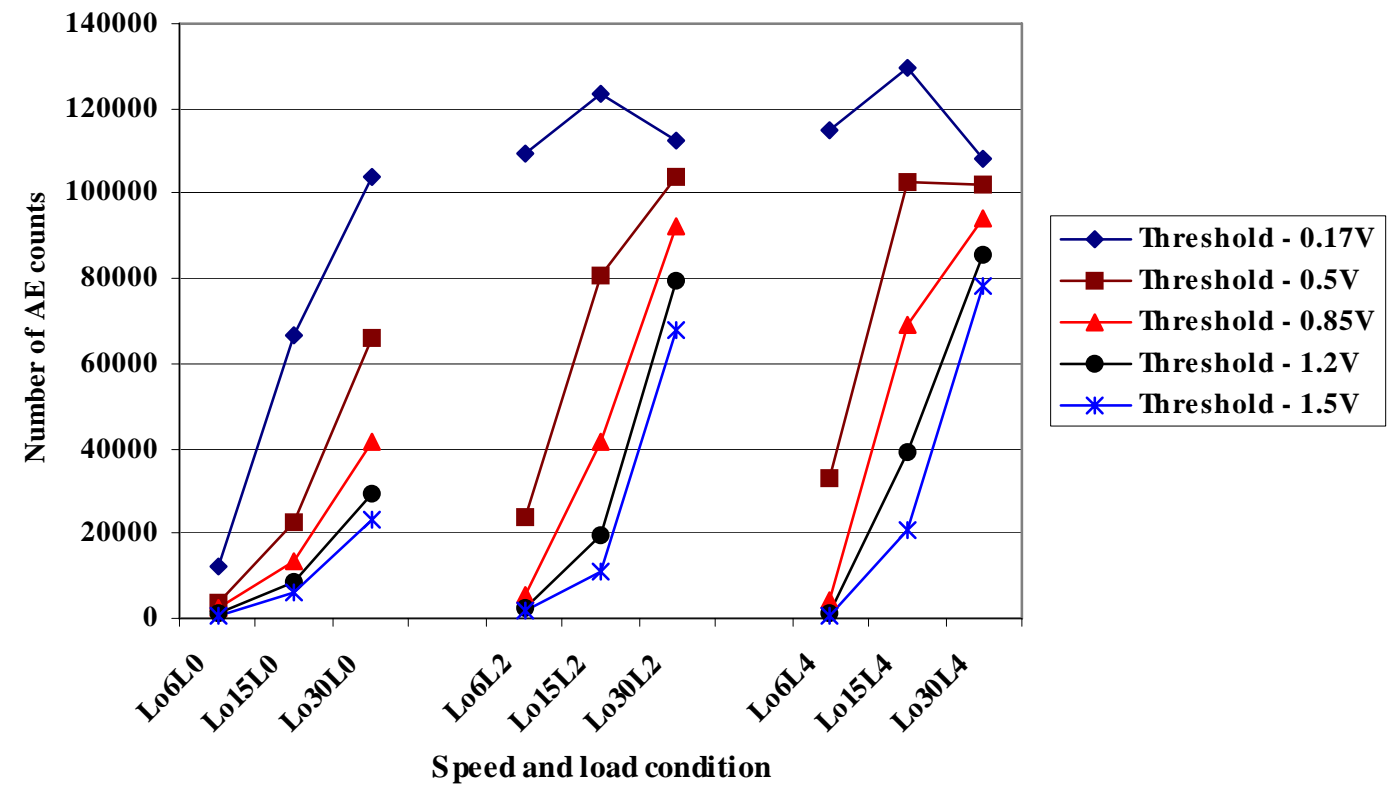

Figure 9 Number of AE counts for a 'large' outer race defect at varying speeds and load conditions 


\section{Discussion}

Background results clearly indicated a rise in r.m.s values with increasing rotational speed. Results from seeded defects indicated than an increase in speed resulted in an increase of r.m.s values. In addition, at fixed rotational speeds there was evidence to suggest that increasing the load also resulted in an increase of r.m.s. This was particularly the case for both 'small' and 'large' outer race defects. For the inner race defect simulation, the same trend was observed for the small defect. However, this was not the case for the inner race line defect though there was an increase in r.m.s values from $0 \mathrm{KN}$ to either $2.4 \mathrm{KN}$ or $4.8 \mathrm{KN}$ but a decrease in from a load of $2.4 \mathrm{KN}$ to 4.8KN, see figure 8 .

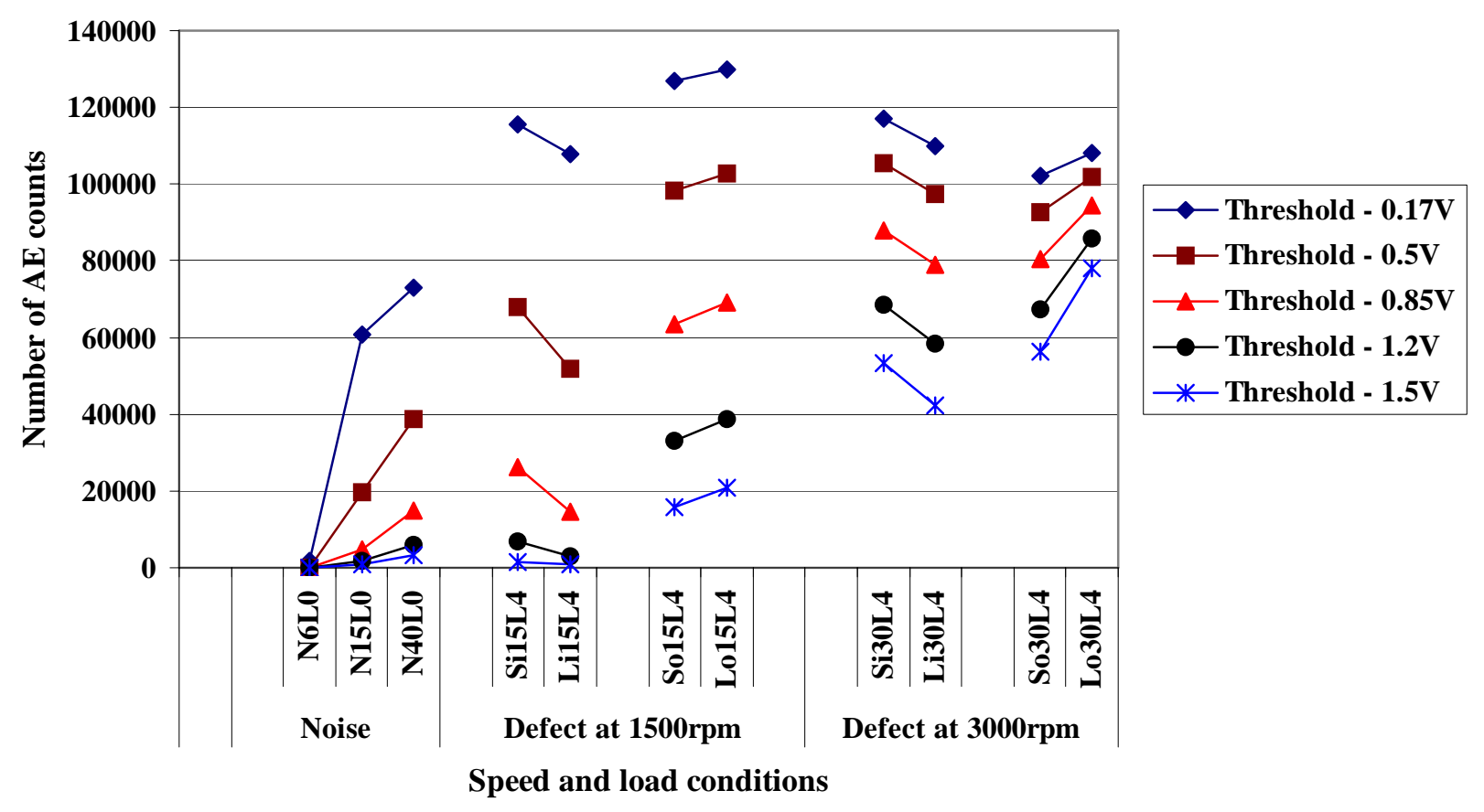

Figure 10 Number of AE counts for a varying speed and load conditions

It was observed that irrespective of the threshold level, there was an increase in $\mathrm{AE}$ counts with outer race defect size. The reverse was observed for inner race defects. However, irrespective of the defect type, there was a clear relationship between an 
increase in counts with speed and load for all threshold levels. It must be noted that whilst the AE counts associated with background noise increased with operational speed, they were still less than all defect conditions, as evident in figure 10. However, counts could not distinguish the types of defects.

\section{Conclusion}

The use of AE parameters such as r.m.s and counts has been validated as a robust technique for detecting bearing damage. In addition, it has been shown that the relationship between bearing mechanical integrity and $\mathrm{AE}$ counts is independent of the chosen threshold level. Whilst numerous exotic diagnostic techniques such as wavelets, higher order statistics, neural networks, etc, could be employed to aid diagnosis, all attempts must be made to keep the method of diagnosis simple and robust as this is the only way to encourage the adoption of this invaluable technique.

\section{References}

Catlin Jr., J.B., (1983), The Use of Ultrasonic Diagnostic Technique to Detect Rolling Element Bearing Defects. Proceedings of Machinery and Vibration Monitoring and Analysis Meeting, Vibration Institute, USA, April 1983, pp 123-130.

Choudhury, A. and Tandon, N (2000), Application of acoustic emission technique for the detection of defects in rolling element bearings, Tribology International, 33, pp39-45.

Mathews, J. R. (1983), Acoustic emission, Gordon and Breach Science Publishers Inc., NewYork. ISSN 0730-7152. 
Olli Yli-Harja, Medial Filters: Extensions, Anaylsis and Design. Lappeenrannan Research Papers, Thesis (Doctor of Technology) - Lappeenranta University of Technology, Paper 13, 1989.

Tan, C C (1990), Application of Acoustic Emission to detection of bearing failures. In the proc. The Inst of Engineers, Australian Tribology conference, Brisbane, Australia, pp 110-114.

Tandon, N. and Nakra, B.C (1990), Defect Detection of Rolling Element Bearings by Acoustic Emission Method, Journal of Acoustic Emission, 1990; 9(1) 25-28. Yoshioka T, Fujiwara T, (1982), New acoustic emission source locating system for the study of rolling contact fatigue, Wear, 81,1, pp183-186.

Yoshioka T, Fujiwara T (1984), Application of acoustic emission technique to detection of rolling bearing failure, American Society of Mechanical Engineers, Production Engineering Division (Publication) PED, 14, pp55-76. 